\title{
On difficult problems and locally graded groups
}

\author{
Olga Macedońska
}

October 21, 2007

\begin{abstract}
Some problems which have a negative answer in general, have an affirmative answer in the class of locally graded groups and a negative answer outside of this class. We present three of such problems and mention another three, which possibly are of that type.
\end{abstract}

Let $\mathcal{F}$ denote the free semigroup with the set of free generators $X=$ $\left\{x_{1}, x_{2}, \ldots\right\}$. Let $u:=u\left(x_{1}, x_{2}, \ldots, x_{n}\right), v:=v\left(x_{1}, x_{2}, \ldots, x_{n}\right)$ be the words in $\mathcal{F}$, that is $u, v$ do not involve inverses of $x_{i}$. We say that a positive (or semigroup) law $u=v$ is satisfied in a group $G$, if under every mapping $\varphi: X \rightarrow G$, it gives an equality $u^{\varphi}=v^{\varphi}$ in $G$.

The notion of locally graded group was introduced by Černikov in 1970 [8].

Definition 1 A group $G$ is called locally graded (LG-group) if every nontrivial finitely generated subgroup in $G$ has a proper subgroup of finite index.

A group which is not an $L G$-group is called a non-LG-group. It is not difficult to prove the following properties of $L G$-groups.

Corollary 1 The class of LG-groups is closed for taking subgroups, extensions and cartesian products; it contains all locally LG-groups and all residually LG-groups.

Examples of $L G$ - and non- $L G$-groups

(i) If $G$ is an extension of a nilpotent group by a locally finite group, then by Corollary $1, G$ is an $L G$-group.

(ii) If $G$ has a finitely generated infinite simple subgroup then $G$ is not an $L G$-group.

2000 Mathematics Subject Classification 20E10, 20F19 
On the picture of Groupland below the class of locally graded groups is shown as the inside shaded ellipse (more details can be found in [15], see also 'grupland' in http://www.google.pl ). The left half of Groupland contains groups without free non-abelian subsemigroups. The right half contains groups with free non-abelian subsemigroups. The north regions $1-4,1^{*}-3^{*}$ contain groups which satisfy no laws. The south-west sector contains groups which satisfy positive laws. The dashed ellipse contains residually finite groups. The region 11 contains groups of polynomial growth. The region 1 contains all known groups of intermediate growth. The non- $L G$-groups are in the regions $4,7,8,3^{*}, 6^{*}$.

Groupland

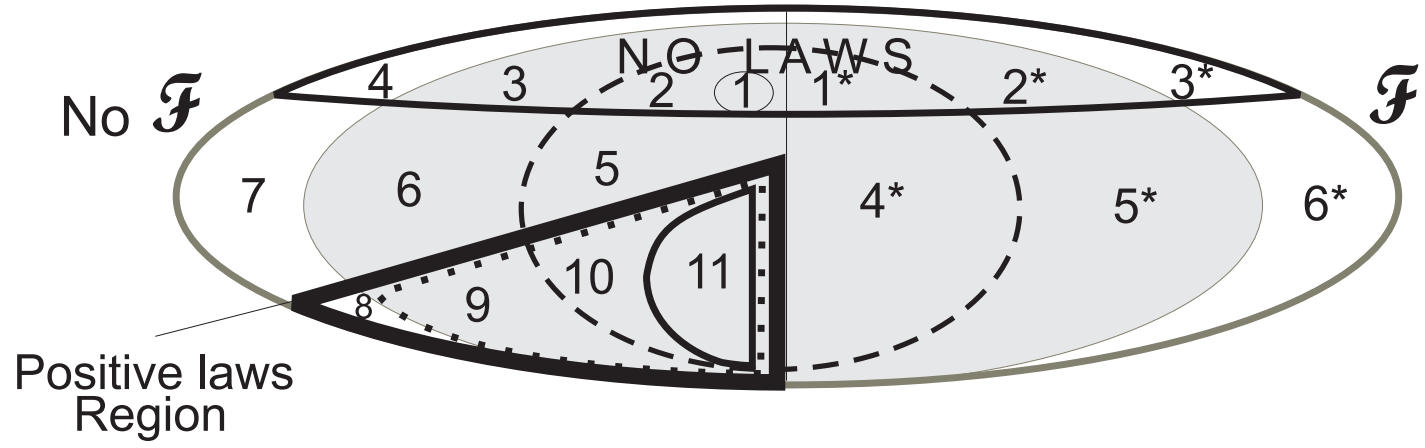

We conjecture that the class of $L G$-groups indicates the difference in methodologies and results between groups built up in standard ways e.g. from soluble and locally finite groups, and those containing finitely generated infinite simple factors. If $G$ is a non- $L G$-group, it must contain a finitely generated infinite simple factor, because if a finitely generated subgroup $H \subseteq G$ has no proper subgroup of finite index, and $N$ is a maximal normal subgroup in $H$ (which exists by Zorn's Lemma), then the factor $H / N$ is simple and infinite.

Some problems which have a negative answer in general, have an affirmative answer for $L G$-groups, and a negative answer for non- $L G$-groups. We start with the Burnside Problem:

\section{P1. Must a group of finite exponent be locally finite?}

Theorem 1 A group $G$ of finite exponent is locally finite if and only if $G$ is an $L G$-group.

Proof Since locally finite groups are locally graded, the 'only if' part is clear. For the 'if' part it suffices to consider a finitely generated locally graded group $G$ of finite exponent. Then from the positive solution of The Restricted Burnside Problem (see [25]), $G$ has a minimal normal subgroup 
$N$ of finite index, which is finitely generated. If $N \neq 1$, then being locally graded, $N$ has a proper subgroup $H$ of finite index. Then $H$ contains a normal subgroup of $G$ of finite index in $G$. This contradicts the minimality of $N$. Hence $N=1$ and $G$ is finite.

By a result of Mal'cev [16] (see also [18]), a group which has a nilpotent subgroup of finite index (is nilpotent-by-finite), satisfies a positive law. The question whether the converse is true was open for more then thirty years. The general answer is "no" [21], however for the class of $L G$-groups the situation is similar to the above.

\section{P2. Must every group satisfying positive laws be nilpotent-by- 'locally finite of finite exponent'?}

Theorem 2 A group $G$ satisfying positive laws is nilpotent-by-'locally finite of finite exponent' if and only if $G$ is an $L G$-group.

Proof The 'only if' part follows by Corollary 1. The 'if' part is proved in ([7] Corollary 1), which says that an $L G$-group which satisfies a positive law must be an extension of a nilpotent group by a locally finite group of finite exponent (see also [2] Theorem 1).

By results of Gromov [9], Milnor [17] and Wolf [26], a finitely generated group has a polynomial growth if and only if it is nilpotent-by-finite. This implies by Theorem 2 the following

Corollary 2 A finitely generated group $G$ satisfying a positive law has a polynomial growth if and only if $G$ is an $L G$-group.

According to the result of Adian [1], the infinite Burnside groups $B(m, n)$ of prime exponent $m \geq 665$ and of a finite rank $n>1$ have exponential growth. Hence by Corollaty 2, these Burnside groups are non- $L G$-groups contained in region 8 of Groupland. The same follows from Theorem 1.

The following problem concerns so called collapsing groups introduced by Shalev in [23]. A group $G$ is called $n$-collapsing if for every $n$-element subset $S \subseteq G$, the inequality $\left|S^{n}\right|<n^{n}$ holds. A group is called collapsing if it is $n$-collapsing for some $n$.

P3. Must every collapsing group be nilpotent-by-'locally finite of finite exponent'?

Theorem 3 A collapsing group $G$ is nilpotent-by-'locally finite of finite exponent' if and only if $G$ is an $L G$-group. 
Proof It is shown in [2], that every collapsing $L G$-group satisfies a positive law, which implies by Theorem 2 , that $G$ is nilpotent-by-'locally finite of finite exponent', and proves the "if" part. The "only if" part follows because nilpotent-by-'locally finite of finite exponent' groups are $L G$-groups.

A group $G$ is called $n$-Engel group if it satisfies the commutator law $[\ldots[[x, y], y], \ldots, y]=1$ where $y$ is repeated $n$ times. In 1963 Shirshov posed the question whether every n-Engel group satisfies a positive law? ([13], 2.82). The question is still open for $n>4$ [24]. However the possible counterexamples must be the non- $L G$-groups.

\section{P4. Must every $n$-Engel group satisfy a positive law?}

Theorem 4 An n-Engel group $G$ satisfies a positive law if $G$ is an LG-group.

Proof Let $\mathfrak{N}_{c}$ denote the variety of nilpotent groups of nilpotency class $c$ and $\mathfrak{B}_{e}$ denote the locally finite variety of exponent $e$ [25]. It is shown by Kim and Rhemtulla [11], that every locally graded $n$-Engel group $G$ is locally nilpotent. Then by a result of Burns and Medvedev [6], $G$ is contained in the variety $\mathfrak{N}_{c(n)} \mathfrak{B}_{e(n)} \cap \mathfrak{B}_{e(n)} \mathfrak{N}_{c(n)}$, where $c(n)$ and $e(n)$ depend on $n$ only. By [16], the variety $\mathfrak{N}_{c(n)} \mathfrak{B}_{e(n)}$ satisfies a positive law, so does $G$.

By $\mathfrak{A}$ we denote the variety of all abelian groups, and by $\mathfrak{A}_{p}$ - the variety all abelian groups of exponent $p$. If a group $G$ satisfies a positive law, then the variety $\operatorname{var}(G)$, it generates, has a basis of positive laws [14]. The variety of all metabelian groups, for example, has no positive laws [16], however each variety contains an abelian subvariety, with the basis of positive laws. By Zorn's Lemma there exist minimal varieties without positive laws, called just not p.l. varieties. The only known just not p.l. varieties are of the form $\mathfrak{A}_{p} \mathfrak{A}$ for prime $p$ [10]. The problem posed in ([22] 19.2), is equiwalent to the following question.

P5. Let $\operatorname{var}(\boldsymbol{G})$ be a variety without positive laws. Does there exist a prime $p$, such that $\operatorname{var}(G) \supseteq \mathfrak{A}_{p} \mathfrak{A}$ ?

Theorem 5 An affirmative answer to P5 is known only for some LG-groups. The problem has a negative answer in general.

Proof An affirmative answer is known for $S C$-groups [10], that is groups which belong to some products of finitely many varieties each of which is either soluble or Cross variety. Since every Cross variety is generated by a finite group [19], it is locally finite, so each $S C$-group is obtained by extensions from soluble and locally finite groups and hence is an $L G$-group. 
The negative answer to the problem is given in [12]. It is shown that there exists a group $G$ which contains a free non-abelian subsemigroup (hence it has no positive laws), while $\operatorname{var}(G)$ does not contain $\mathfrak{A}_{p} \mathfrak{A}$ for any $p$. This group $G$ is the finitely generated relatively free group, which is studied in Chapter 9 of [20]. It defines a pseudo-abelian variety in which all metabelian and all finite groups are abelian. However it is not known whether $G$ is a non- $L G$-group.

The following problem was posed in 1981 by G. Bergman [3],[4], and can be found in a different form also in [22].

P6. Must every group $G$ satisfy all of the laws holding in its generating subsemigroup $S$ ?

The affirmative answer is known if $G$ is an $L G$-group without free nonabelian subsemigroups (not published). The existence of a counterexample was announced by S. Ivanov and A. Storozhev at the Algebraic Conference in Moscow 2004. Their semigroup $S$ satisfies a law similar to that defined in [21]. The relatively free group with that law, constructed in [21], is a non$L G$-group. However it is not known whether a group $G$ is a non- $L G$-group if only its generating subsemigroup $S$, satisfies that law.

We summarize the above information in the following table.

\begin{tabular}{|c|c||c|}
\hline Problem & an answer for $L G$-groups & an answer for non- $L G$-groups \\
\hline \hline P1. & $\forall G$, YES & $\forall G, \mathrm{NO}$ \\
\hline P2. & $\forall G$, YES & $\forall G$, NO \\
\hline P3. & $\forall G$, YES & $\forall G$, NO \\
\hline P4. & $\forall G$, YES & $\exists G, \mathrm{NO}$ (is $G$ non- $L G$-group?) \\
\hline P5. & $\exists G$, YES & $\exists G, \mathrm{NO}$ (is $G$ non- $L G$-group?) \\
\hline P6. & $\exists G$, YES & \\
\hline
\end{tabular}

\section{References}

[1] S. I. Adian, The problem of Burnside and identities in groups, Nauka, Moscow, 1975. (Russian) (see also, trans. J.Lennox and J.Wiegold, Ergebnisse der Mathematik und ihrer Grenzgebiete 92, Springer-Verlag, Berlin, 1979).

[2] B. Bajorska, O. Macedośka, On positive law problems in the class of locally graded groups, Communications in Algebra, 32(5) (2004), 18411846 .

[3] G. Bergman, Hyperidentities of groups and semigroups, Aequat. Math. 23 (1981), 55-65. 
[4] G. Bergman, Questions in algebra, Preprint, Berkeley, U.C. 1986.

[5] M. Boffa, Elimination of inverses in groups, Model Theory of Groups and Automorphism Groups, London Math. Soc. Lecture Notes Series, 224 (1997), 134-143.

[6] R. G. Burns, Yu. Medvedev, A note on Engel groups and local nilpotence, J. Aust. Math. Soc. 64 (1998), 92-100.

[7] Robert G. Burns, Yuri Medvedev, Group Laws Implying Virtual Nilpotence, J. Aust. Math. Soc. 74 (2003), 295-312.

[8] Černikov, Infinite nonabelian groups with an invariance condition for infinite nonabelian subgroups (Russian), Dokl. Akad. Nauk SSSR 194 (1970), 1280-1283.

[9] M. Gromov, Groups of polynomial growth and expanding maps, Publs. Math. Inst. hautes étud. sci. 53 (1981), 53-73.

[10] J. R. J. Groves, Varieties of soluble groups and a dichotomy of P. Hall, Bull. Austral. Math. Soc. 5 (1971), 391-410.

[11] Y. Kim, A. H. Rhemtulla, On locally graded groups. Groups-Korea'94, de Gruyter, Berlin, 1995, 189-197.

[12] P. Kozhevnikov, O. Macedońska, On varieties of groups without positive laws, Communications in Algebra 30(9) (2002), 4331-4334.

[13] The Kourovka Notebook: unsolved problems in group theory, 12th ed., Inst. Math. Sibirsk. Otdel. Akad. Nauk Rossii, Novosibirsk, 1993.

[14] Jacques Lewin and Tekla Lewin, Semigroup laws in varieties of soluble groups, Proc. Camb. Phil. Soc. 65 (1969), 1-9.

[15] O. Macedońska, Groupland, Groups St Andrews 2001 in Oxford, Eds.: C. Campbell, E. Robertson, G. Smith, Cambridge University Press (2003), 400-404.

[16] A. I. Mal'cev, Nilpotent semigroups, Uchen. Zap. Ivanovsk. Ped. Inst. 4 (1953), $10^{7}-111$.

[17] J. Milnor, Growth of finitely generated solvable groups, J. Differential Geometry 2 (1968), 447-449.

[18] B. H. Neumann and Tekla Taylor, Subsemigroups of nilpotent groups, Proc. Roy. Soc. Ser. A 274 (1963), 1-4. 
[19] S. Oates, M. B. Powell, Identical relations in finite groups, J. Algebra 1 (1964), 11-39.

[20] A. Yu. Ol'shanskii, Geometry of defining relations in groups; Mathematics and its applications (Soviet Series), 70; Kluwer Academic Publishers: Dordrecht, 1991.

[21] A. Yu. Ol'shanskii and A. Storozhev, A group variety defined by a semigroup law, J. Austral. Math. Soc. (Series A), 60 (1996), 255-259.

[22] L. N. Shevrin, M. V. Volkov, Semigroup identities (Russian), Izv. Vuzov, Matematika 11 (1985), 3-4\%.

[23] A. Shalev, Combinatorial conditions in residually finite groups, II, J.Algebra 157 (1993), 51-62.

[24] G. Traustason, Semigroup identities in 4-Engel groups, J. Group Theory, 2 (1999), 39-46.

[25] M. Vaughan-Lee, On Zelmanov's solution of the restricted Burnside problem, J. Group Theory, 1 (1998), 65-94.

[26] J. A. Wolf, Growth of finitely generated solvable groups and curvature of Riemannian manifolds, J. Differential Geometry 2 (1968), 421-446. 